\title{
The Metaphysics of Emergent Spacetime Theories
}

\author{
Niels C.M. Martens* \\ This is the Accepted Version, i.e. post-peer-review but pre-type-setting, of \\ "Martens, N.C.M. (2019), The Metaphysics of Emergent Spacetime Theories. \\ Philosophy Compass, 14(7):e12596, https://doi.org/10.1111/phc3.12596"
}

\begin{abstract}
The debate concerning the ontological status of spacetime is standardly construed as a dilemma between substantivalism and relationalism. I argue that a trilemma is more appropriate, emergent spacetime theories being the third category. Traditional philosophical arguments do not distinguish between emergent spacetime and substantivalism. It is arguments from physics that suggest giving up substantivalism in favour of emergent spacetime theories. The remaining new dilemma is between emergent spacetime and relationalism. I provide a list of questions, which one should consider when discussing emergent spacetime theories, and apply them to a quantum superfluid toy model of emergent spacetime.
\end{abstract}

Keywords -Emergent spacetime, substantivalism, relationalism

\section{A third candidate: emergent spacetime}

The debate concerning the ontological status of spacetime is usually portrayed as a dilemma, namely substantivalism vs. relationalism. ${ }^{1}$ This would suggest that substantivalism claims a single thesis $T_{1}$ to be correct, with relationalism asserting $\neg T_{1}$. $T_{1}$ is commonly taken to be that 'spacetime is primitive (i.e. fundamental)' (Lehmkuhl, 2018; Pooley, book manuscript). ${ }^{2}$ However, relationalism does not merely hold that 'spacetime is derivative (i.e. non-fundamental)'

\footnotetext{
${ }^{*}$ RWTH Aachen University, Institute for Theoretical Particle Physics and Cosmology, Sommerfeldstrasse 16, 52074 Aachen, Germany, martens@physik.rwth-aachen.de, +49 241 80 27054, Orcid:0000-0002-2839-1387

${ }^{1}$ See Pooley (2013) and Dasgupta (2015) for some recent, accessible introductions to the substantivalism-relationalism debate. The locus classicus is the Leibniz-Clarke correspondence (Alexander, 1956/1717).

${ }^{2}$ Alternatively, substantivalism is sometimes construed as the combination of a thesis $T_{1}^{\prime}$ that space(time) and its parts are real and a second thesis $T_{2}^{\prime}$ that those real parts are basic/primitive/fundamental constitutents of our world. Relationalism is then portrayed as denying the reality of space(time) (arguably by Leibniz himself (Alexander, 1956/1717)). Such a portrayal of the debate is misleading. (Pooley's title of his forthcoming book on the
} 
$\left(\neg T_{1}\right)$, but adds that spacetime is derivative from matter. This leaves open the option that spacetime could be derived from other (fundamental) degrees of freedom. ${ }^{3}$ This view is dubbed emergent spacetime (Carlip, 2012; Matsubara, 2017); examples within this research programme include spacetime emerging from (bosonic) strings, from quantum graphs (Konopka et al., 2006, 2008), from condensed matter systems (Bain, 2013), from causal sets (Dowker, 2008) and from spin networks (Rovelli and Vidotto, 2014). ${ }^{4}$ The debate is thus minimally a trilemma.

All this suggests that the focus should not only be on the status of spacetime (ST), but also on the status of matter (M). Let us introduce a second thesis $T_{2}$ : 'ST \& M are ontologically distinct'. That is, ST \& M do not ontologically depend on each other in any way. ${ }^{5} \neg T_{2}$ is true iff there is any ontological connection at all between ST \& M, whether that is a relation of identity, supervenience,

substantivalism-relationalism debate, The Reality of Spacetime (Pooley, book manuscript), seems to suggest a similar misleading definition of relationalism, but the contents in fact agree with the definitions in this paper.) First of all, $T_{1}^{\prime}$ (reality) is implied by $T_{2}^{\prime}$ (primitiveness) and thus redundant. Secondly, at least in the modern debate, relationalism holds that space is derivable from relational, spatiotemporal properties of matter. Hence, space is as real as the relational properties are, it is just not fundamental (Belot, 2011). As an analogy, consider a chair from the perspective of a particle physicist. The particle physicist would not deny that the chair is real, but would deny that it is fundamental. The chair is made up of equally real, but more fundamental elementary particles (or fields, or strings, etc.). All spacetime theories in this paper consider space(time) to be real; fundamentality is the issue, not reality (North, 2017).

${ }^{3}$ If neither material nor spatiotemporal, how else could these degrees of freedom be qualified? What I mean by spacetime (matter) is that whatever one usually takes to be the complete set of properties of spacetime (matter), such as topological structure, $3+1$ dimensions, light-cone structure (the appropriate dynamics), etc. (see also the end of Section 1 in the main text). The other degrees of freedom will not have all of these properties, although they might typically have some (cf. Le Bihan and Linnemann (2018)) or many of these (although sometimes none at all, or these properties are not even coherent: for a huge quantum graph (see main text) with many criss-cross connections, it is meaningless to attribute any notion of dimensionality or topology at all). It may then be tempting to dub these other degrees of freedom 'proto-spacetime', or 'matter-like degrees of freedom', instead of a wholly novel name. This is mere semantics, and I will not dwell on it here. The point is that the other degrees of freedom do not have the complete set of properties of either spacetime or matter, and hence belong to a third category. The main text refers to various examples of other degrees of freedom, with varying degrees of similarity to matter or spacetime proper.

${ }^{4}$ Sometimes some of these theories are interpreted as vindicating relationalism. Such discussions still operate under the mistaken assumption that the substantivalism-relationalism distinction is an exhaustive dichotomy. They claim that, on these theories, spacetime is not fundamental and take this to imply that relationalism is true. All this does imply is that spacetime is derivative from relations between relata that are not spacetime points. Logically, this leaves open that the relata could be either matter or other degrees of freedom that are neither (fully) matter nor (fully) spatiotemporal. Neither causal sets, nor spin networks, for example, are (fully; cf. fn.3) matter; hence, with spacetime not being fundamental in these theories, they are, using the terminology of this paper, emergent spacetime theories rather than relational theories. They would only be relational theories if relationalism were to be redefined as the claim that the relata of spatioremporal relations are not spacetime pointswhich would conflate my narrower definition of relationalism with emergent spacetime.

${ }^{5}$ But see Lehmkuhl (2011) for a large variety of ways in which matter does depend on the metric in general relativity. It is outside the scope of this paper to discuss to what extent those dependencies are forms of ontological dependence. 


\begin{tabular}{c|cc} 
& $T_{2}$ & $\neg T_{2}$ \\
& $\mathrm{ST} \& \mathrm{M}$ are distinct & ST \& M are not distinct \\
\hline$T_{1}:$ ST is primitive & dualistic substantivalism & supersubstantivalism \\
$\neg T_{1}: \mathrm{ST}$ is derivative & (dualistic) emergent ST & relationalism
\end{tabular}

Table 1: Matrix of views on spacetime (ST) and matter (M)

grounding, constitution, 'property of', 'excitation of', 'stable geometric pattern of', or anything else. ${ }^{6}$ We will not debate the comparative merits of these options here. One could prima facie agree or disagree with $T_{1}$ and $T_{2}$ independently, resulting in a $2 \times 2$ matrix of categories, see Matrix 1 . The term 'supersubstantivalism' in this matrix is the popular name for monistic substantivalism, the view that ST is the only primitive substance or entity. The ontological relation of $\mathrm{M}$ to primitive ST could be cashed out in many ways, but as mentioned before this paper is not the place to take a stance on this issue. Discussions of substantivalism in the literature often implicitly assume dualistic substantivalism, by implicitly adding $T_{2}$ to $T_{1}$. This suggests that emergentists and dualistic substantivalists have a lot of common ground, the former providing a constructive ${ }^{7}$ theory for the latter. For some it may even seem that the spirit of substantivalism is mainly contained within $T_{2}$, in which case emergent spacetime perhaps deserves the name 'substantivalism' more than supersubstantivalism. As this is merely an issue of terminology I will not dwell on it here. Common ground may similarly be found between supersubstantivalists and relationalists, which make up the right half of the matrix.

By assigning emergent spacetime to the lower left corner of Matrix 1 only, we have implicitly assumed a dualistic version of emergent spacetime, which holds that spacetime emerges from more fundamental degrees of freedom, and that matter is a distinct entity that lives within that emergent spacetime. One may then ask what would happen to that matter in the (energy) regime where spacetime does not emerge. It seems strange to hold on to a 'containee' once the container is gone. To avoid this worry we could either opt for some sort of superemergent spacetime $^{8}$, where spacetime is derivative from lower level degrees of freedom and matter is in turn ontologically linked to that emergent spacetime (in the regime where spacetime emerges only, of course), or for emergent spacetime \& matter, where both ST and M emerge directly from the fundamental degrees of freedom. Alternatively, we could hold on to dualistic emergent spacetime and face the issue head-on by extending our notion of matter to something that can 'live on the structure of the fundamental degrees of freedom', even in the regime where that structure does not resemble spacetime as we know it. For instance, in the case of quantum graphity (Konopka et al., 2006, 2008),

\footnotetext{
${ }^{6}$ See Schaffer (2009) and Lehmkuhl (2009) for some examples.

${ }^{7} \mathrm{I}$ have adopted this terminology from Einstein (1919).

${ }^{8}$ I prefer this terminology over emergent superspace(time), as superspace is already associated both with supersymmetry and Wheeler's superspace, and over emergent supersubstantivalism, as the core commitment of any substantivalist view is that spacetime is primitive (Lehmkuhl, 2018).
} 


\begin{tabular}{c||c|c} 
& $\begin{array}{c}T_{2} \\
\text { ST \& M are distinct }\end{array}$ & $\begin{array}{c}\neg T_{2} \\
\text { ST \& M are not distinct }\end{array}$ \\
\hline \hline$T_{1}$ : ST is primitive & dualistic substantivalism & supersubstantivalism \\
\hline$\neg T_{1}$ : ST is derivative & $\begin{array}{c}\text { dualistic emergent ST } \\
\text { (emergent ST \& M ?) }\end{array}$ & $\begin{array}{c}\text { relationalism } \\
\text { super-emergent ST } \\
\text { (emergent ST \& M ?) }\end{array}$
\end{tabular}

Table 2: Updated matrix of views on spacetime (ST) and matter (M)

\begin{tabular}{c||c|c} 
& $T_{3}$ & $\neg T_{3}$ \\
& $\mathrm{M}$ is primitive & $\mathrm{M}$ is derivative \\
\hline \hline$T_{1}:$ ST is primitive & dualistic substantivalism & supersubstantivalism \\
\hline$\neg T_{1}$ : ST is derivative & dualistic emergent $\mathbf{S T}$ & emergent $\mathbf{S T} \& \mathbf{M}$ \\
& relationalism & super-emergent $\mathbf{S T}$
\end{tabular}

Table 3: Alternative matrix of views on spacetime (ST) and matter (M)

'matter' is represented by a quantized Hamiltonian placed on a graph. Only in the low energy regime are the correct edges 'switched on' for the topology of the vertices to reduce to a four-dimensional manifold. Outside that regime one could still talk about matter as the solution to the Hamiltonian which lives on the edges, even if the number of edges connected to each vertex exceeds the amount required for $((3+1)$-dimensional $)$ spacetime to emerge. Similar stories apply in tensor models and group field theory. ${ }^{9}$

Regardless of whether one is convinced of the coherence of this construction of dualistic emergent spacetime, the other two categories remain prima facie available. The corresponding updated version of our matrix of categories is Matrix 2. In which category 'emergent ST \& M' belongs depends on the details of the theory. Can spacetime and matter emerge independently, or are they dependent in such a way that an equivalent super-emergent ST theory could be constructed? If it turns out that the majority of emergent spacetime theories is not the group of dualistic emergent spacetime theories, a different categorisation may be more useful. After all, we would like a categorisation that splits up the main four contenders (dualistic substantivalism, supersubstantivalism, relationalism and the dominant form of emergent spacetime (and matter)). One such categorisation, represented by Matrix 3, additionally solves the difficulties with categorising 'emergent ST \& M'. It replaces $T_{2}$, which compared spacetime with matter, with $T_{3}$ which focuses solely on the status of matter: 'matter is primitive'.

It is now only a small step to combine all three theses into a single matrix, such that all six types of spacetime \& matter theories are in different cells. Merging the previous two matrices we obtain Matrix 4. Here we have assumed for the emergent spacetime and matter theories (EST\&M) that matter emerges from the more fundamental degrees of freedom independently of the emergent

\footnotetext{
${ }^{9}$ I would like to thank an anonymous referee for pointing this out.
} 


\begin{tabular}{|c|c|c|c|}
\hline & & $\begin{array}{c}T_{3} \\
\mathrm{M} \text { is primitive }\end{array}$ & $\begin{array}{c}\neg T_{3} \\
\mathrm{M} \text { is derivative }\end{array}$ \\
\hline \multicolumn{2}{|l|}{$T_{1}: \mathrm{ST}$ is primitive } & "Dual Subst & SupSubst \\
\hline \multirow{2}{*}{$\neg T_{1}: \mathrm{ST}$ is derivative } & $T_{2}: \mathrm{ST} \& \mathrm{M}$ are distinct & Dual EST & EST\&M \\
\hline & $\neg T_{2}$ : ST \& M are not distinct & Rel & SupEST \\
\hline
\end{tabular}

Table 4: Final matrix of views on spacetime (ST) and matter (M)

spacetime. When this is not the case such a view is to be categorised as a super-emergent ST theory. ${ }^{10}$

Our final result, Matrix 4, provides us with a more comprehensive, exhaustive and rigorous map of the space of logical possibilities than was provided by just the orthodox substantivalism-relationalism distinction.

Finally, note that for any of the above views to be well-defined, it is crucial to define spacetime and matter properly. Unfortunately this distinction is usually considered to be so basic and intuitive that many authors do not feel the need to be explicit about what this distinction is supposed to be. ${ }^{11}$ However, that this distinction is far from trivial becomes especially clear when we ask the controversial question of whether the metric/gravitational field in general relativity (GR) is considered to be part of spacetime or of matter (or both, or neither) (Hoefer, 1996). On the one hand it encodes the geometric and hence causal structure of spacetime: without such structure a manifold can hardly be said to represent spacetime (Maudlin, 1988). On the other hand, in GR it becomes a dynamical field satisfying an action-reaction principle and containing gravitational waves that carry energy (Earman and Norton, 1987; Read, 2018) (pace Hoefer (1996) and Dürr (2015)). In this sense it seems on a par with matter fields. However, matter fields can be null over finite regions of the manifold, whereas the metric field cannot (Hoefer, 1996). One may respond to this that being null over finite regions does not imply that it does not exist (in those regions): the Higgs field, for instance, has a non-minimal energy when

\footnotetext{
${ }^{10}$ If desired, it would be possible to hold on to a distinction between theories in which matter is directly derivable from spacetime ('super' theories), and theories in which spacetime and matter do depend on each other through the more fundamental degrees of freedom but in which matter is not directly derivable from spacetime. Whereas the first three theses concerned the ontology of entities, the required $T_{4}$ concerns properties. Is matter a geometrical property, or is it a fundamental, distinct property of the underlying degrees of freedom (either spacetime points or more fundamental degrees of freedom)? If we formulate $T_{4}$ as 'matter is a geometrical property', it can be used to subdivide the ' $\neg T_{3}$ column' of our matrix. This not only distinguishes the two types of EST\&M theories that we were after, but also follows the popular distinction in the literature between 'super' theories which reduce the matter degrees of freedom to geometrical degrees of freedom-e.g. Wheeler (Misner et al., 1973) and Lehmkuhl (2009, 2018) — and 'super' theories which hold that spacetime points or the more fundamental degrees of freedom have two properties: geometrical and material (Schaffer, 2009). Lehmkuhl dubs these positions radical and modest supersubstantivalism, respectively.

${ }^{11} \mathrm{~A}$ positive exception is the recent debate on spacetime functionalism, which is the view that 'to be spacetime is to play the spacetime role'. This literature does, of necessity, discuss in detail what the spacetime role or concept is supposed to be (Baker, 2018; Knox, 2013, 2018).
} 
it takes on a null value (Maudlin, 1993). Here I will not take a stance on this issue, but merely point out that it needs to be decided before the positions in this paper will be well-defined. ${ }^{12}$ Another important issue concerns whether primitive identities of spacetime points are to be included in spacetime (Hoefer, 1996; Pooley, 2002, book manuscript) - or perhaps spacetime points themselves should be eliminated altogether (as per relationalism). I will return to this issue in Section 2.1.

\section{The new dilemma: emergent spacetime vs. sub- stantivalism}

In the previous section is was argued that 'emergent spacetime' is an important third candidate in the debate concerning the ontological status of spacetime (and matter). The emergent spacetime research programme is the focus of (the remainder of) this paper; this section will continue our discussion of how it relates to the other two candidates, whereas the subsequent two sections will focus solely on the emergent spacetime research programme. In this section I will make plausible that, insofar as substantivalism is an effective theory of emergent ST, the strongest philosophical arguments against substantivalism do not distinguish between substantivalism and emergent spacetime theories. It is the arguments from physics that favour emergent spacetime over substantivalism. Hence, the philosophical debate should focus on the remaining dilemma between emergent spacetime theories and relationalism. The subsequent section continues by listing some questions that we would like the emergent spacetime research programme to answer, followed by a section that applies those to a quantum superfluid toy model in which spacetime emerges.

\subsection{Philosophical arguments}

Perhaps the strongest philosophical argument in the classical debate between relationalism and substantivalism which favours relationalism is what is now called the (static) Leibniz shift argument (Alexander, 1956/1717; Pooley, book manuscript). Consider a possible world that is a solution to Newtonian gravity (NG). If space were to exist as a primitive substance, one could generate infinitely many metaphysically distinct possible worlds by Leibniz shifting this original solution, that is by uniform translations of all matter relative to the background space. Since the laws of Newtonian gravity only care about distances between material bodies and not about their absolute positions, all these

\footnotetext{
${ }^{12}$ (Further) skepticism concerning the tenability of a clear spacetime-matter dichotomy may arise from Einstein's views on the aether in General Relativity (Rynasiewicz, 1996), electrodynamical theory at the close of the nineteenth century (Rynasiewicz, 1996), the AdS/CFT duality between one theory that prima facie looks like a theory of matter and another that prima facie looks like a theory of gravity/spacetime (Le Bihan and Read, forthcoming), and the dark matter vs. modified gravity debate (Martens and Lehmkuhl, manuscript).
} 
worlds related by Leibniz shifts are empirically equivalent solutions to NG. Substantivalism thus generates metaphysical distinctions without an empirical difference. It is considered sound methodology - namely a specific instance of an Occamist norm, called the Principle of Identity of Indiscernibles - to avoid such distinctions when possible.

This argument concerns the physical relation between space and matter, namely their relative positions, and not the absolute fundamentality of spacetime itself - it bears (at best) upon the relative fundamentality between spacetime and matter only. Hence, insofar as a dualistic 'substantivalist' theory is an effective theory of another dualistic emergent spacetime theory, or a 'supersubstantivalist' theory an effective theory of another super-emergent spacetime theory, the argument doesn't distinguish between them, at least not in the regime where spacetime emerges. Outside this regime it is unlikely that one could even formulate the static Leibniz shift argument. Similarly, it is unclear how one could formulate the argument against a theory of emergent spacetime $\&$ matter, without knowing the details of such a theory.

A popular version of substantivalism that dissolves this argument (and simultaneously its relativistic brother the hole argument (Earman and Norton, 1987)) is sophisticated substantivalism (Hoefer, 1996; Pooley, 2002, book manuscript). It is often assumed that a substantivalist must accept an infinity of distinct yet qualitatively indiscernible worlds related by Leibniz shifts. However, substantivalism is merely a thesis about spacetime being mentioned on the list of fundamental things in the ontology of our actual world. There is no need to commit to claims about transworld-identity of parts of space(time). Without such primitive identities of space(time) points, there is no way to distinguish all the possible worlds related by a Leibniz shift. All such putative worlds collapse to one: the actual world. Hence, insofar as space(time) prima facie emerges without primitive identities of space(time) points in theories of (super)emergent spacetime - i.e. sophisticated space(time) emerges - these theories can equally well avoid the static Leibniz shift argument and hole argument.

A second classical argument against substantivalism in its original form, the kinematic shift argument, targets the invariance of Newtonian physics under a uniform velocity shift, that is the Galilean relativity principle. Once again the substantivalist seems committed to a plurality of physically possible yet observationally indiscernible worlds. Sophisticated substantivalism is of no avail here, since the different worlds - although observationally indistinguishableare not qualitatively indistinguishable (pace Dewar (forthcoming) $)^{13}$. Whereas body $B$ may be at rest with respect to region $R$ in the actual world, there is no region $R^{\prime}$ in the kinematically shifted world that is at rest with respect to body $B$. However, this argument is commonly evaded by replacing Newtonian space and time with Neo-Newtonian (also known as Galilean) spacetime. As we only consider theories of emergent spacetime, these automatically evade the kinematic shift argument. Further discussions of minor arguments in favour of relationalism are provided by Pooley (book manuscript, 2013), but seem not to

\footnotetext{
${ }^{13}$ See Martens and Read (manuscript) for a critical response to Dewar.
} 
form any specific problem for emergent spacetime theories.

In fact, in response to one specific argument - which is problematic for relationalism but can be dealt with by substantivalism when it is slightly modifiedemergent spacetime theories may be even more successful than their effective, substantivalist counterparts by naturally providing the required modification. Several authors argue that parity violating laws of nature can only be explained if spacetime has an orientational structure (Arntzenius, 2012; Earman, 1989; Huggett, 2000; Martens, 2012; Pooley, 2003). It is then furthermore argued that the relationalist cannot provide such a structure, whereas the substantivalist could postulate that absolute spacetime has this additional structure. If spacetime instead emerges with a ready-made orientation, providing a dynamical explanation for this orientation rather than having to postulate it, this would be a strong point in favour of emergent spacetime.

\subsection{Arguments from Physics}

We have seen in the previous subsection that (unless spacetime emerges with primitive identities) emergent spacetime theories fare as well, if not better, against philosophical arguments that target substantivalism. In this subsection I will discuss arguments from physics that suggest that we should favour emergent spacetime theories over their effective counterparts (i.e. substantivalism).

Arkani-Hamed (2010) and Seiberg (2005) $)^{14}$ argue that the concept of spacetime becomes useless at small length scales, as is often voiced by Arkani-Hamed's famous slogan ' $Q M+$ gravity = spacetime is doomed'. They argue that, due to Heisenberg's uncertainty principle, we need to use an energy $E>1 / r$ to probe distances of size $r$. Without gravity there is no problem doing this, at least in principle (but see Hossenfelder (2013)). However, when gravity is turned on the concentration of energy required to probe distances below the Planck scale collapses into a black hole. This singularity precludes an operational definition of distances smaller than the Planck length (and times smaller than $\left.\sim 10^{-43} \mathrm{~s}\right)^{15}$. Arkani-Hamed adds to this that the size of the particle accelerator required to probe such energies would cause the apparatus itself to collapse into a black hole. He reminds us that every time we have encountered quantities or ideas in the past that one cannot even in principle associate some operational meaning with, it always turned out that those ideas were approximate and emerge from something more fundamental.

\footnotetext{
${ }^{14}$ Ideas in this vicinity can be traced back to Bronstein (Bronstein, 1936; Gorelik, 2005), Snyder (1947), Peres and Rosen (1960), Mead $(1964,1966)$ and many others, and have received significant attention through Doplicher et al. (1995). See also Hossenfelder (2013), Rovelli and Vidotto (2014) and Matsubara (2017).

${ }^{15}$ In Wüthrich's (2005) interpretation of Snyder's (1947) and Doplicher et al.'s (1995) arguments, the operational definition of these distances is not possible because the created black hole prevents signals from escaping and completing the measurement. However, even if the measurement could (per impossibile) be completed, the problem would remain, because spacetime would have been destroyed, and it would not be clear what the signal is supposed to be a measurement of.
} 
Arkani-Hamed cautions us to not conclude from this that there are atoms of space(time). (Although this would not be a problem for the toy model discussed below, or the abovementioned Quantum Graphity, it would prima facie seem to be a problem for causal set theory which takes spacetime to be discrete (Dowker, 2008) and for spin networks (Rovelli and Vidotto, 2014).) The problem with spacetime is not only a localised problem; there is no concept of spacetime at all. This seems strange: General Relativity has been an extremely useful, if not perfect tool at length scales larger than the Planck scale. Surely we expect any fundamental theory replacing General Relativity to generate General Relativity as an effective theory at large length scales. Arkani-Hamed's argument why it cannot be true that there is new physics only below the Planck scale, i.e. at sizes smaller than the supposed atoms of spacetime, is that this would go against relativity. Scales are not absolute, but depend on one's reference frame. Hence, we would get new physics in one reference frame, but not in another Lorentz-boosted reference frame. In other words, Arkani-Hamed argues, there is no Lorentz covariant way to define atoms of spacetime. Here I think it is important to remember what we are after. We want an operational definition of length below the Planck scale. We never required lengths to be Lorentz invariant, so why would we require atoms to be? Why could not spacetime emerge in one reference frame, whereas in the other we observe the discrete structure of spacetime? After all, it is very common to deal with the non-renormalisability of quantum field theories by imposing a high energy/small length cut-off, despite this cut-off of course not being Lorentz covariant either. ${ }^{16}$ But perhaps this analogy only makes salient that this cut-off is equally problematic. In this case we have a deep problem to face, even before attempting to marry quantum theory with gravity - a problem that I do not have the answer to. An alternative route that one might take to avoid this whole problem is by considering theories where Lorentz invariance breaks down before the discreteness sets in, or by deforming Lorentz invariance as to allow an extra invariant scale (Amelino-Camelia, 2002). In a similar vein, the case study in Section 4 will show that it is possible to get an emergent Lorentz-covariant spacetime from a fundamental non-relativistic spacetime (which in that example is continous though). Furthermore ${ }^{17}$, causal set theory has found a way to retain local Lorentz invariance (in the continuum approximation) despite its atomic nature, by imposing a stochastic dynamics for generating the causal sets which itself is invariant under Lorentz transformations (even though the created causal sets of course still break Lorentz invariance at the scale where their discreteness becomes relevant).

Returning to Arkani-Hamed and Seiberg's first point that spacetime is doomed, it should be pointed out that this is an epistemological statement: when probing spacetime at small length scales we destroy it, which makes it impossible for us to know anything about spacetime at such scales. This is not very surprising or novel - we are very used to measurements disturbing the system, eg. in regular quantum physics. More importantly, in this paper we are interested in

\footnotetext{
${ }^{16}$ See also Donoghue (2012).

${ }^{17}$ See Hossenfelder's excellent review (2013) for a discussion of alternatives.
} 
the ontology of spacetime. We cannot leap from the epistemological problem of not being able to know the local structure of spacetime to the ontological claim that there is no such thing - doesn't the argument that something gets destroyed in fact admit that there was something to begin with? One might argue that an Occamist principle - do not multiply entities beyond necessity could close the gap between epistemology and ontology, but it is simply not clear in this context that the non-emergentist is postulating any extra, unnecessary entities. Spacetime is one big entity; all scales come as a package deal. It seems to be a category mistake to treat the small scales as extra entities that we could just throw out. In fact, isn't it the emergentist who is postulating new entities? Thus, I suggest we hold our horses and refrain from giving in to Arkani-Hamed's operationalist urge to conclude, merely from the epistemological argument that we destroy spacetime when probing it at small scales, that there never was any spacetime at those scales to begin with.

An ontological counterpart of the previous story is however suggested by Wheeler (Misner et al., 1973). He reminds us that empty space is not empty: the vacuum is the seat of the most violent physics we can imagine; macrophysics is nothing compared to it. At small enough distances the Heisenberg uncertainty principle allows creation of particle-antiparticle pairs out of the vacuum. Combining this with General Relativity, which tells us that the mass and energy of those pairs curve the local spacetime, spacetime becomes very irregular at small scales, although it remains smooth at large scales. It is sometimes argued that this already shows that General Relativity, which in its standard form takes spacetime to be smooth at all scales, is not valid at the Planck scale and is only an effective theory above that scale. However, up till here it seems to me that there is no problem with incorporating quantum fluctuations within General Relativity. Relativists assumed spacetime to be smooth at small scales, since they were not aware that at those scales there is a huge amount of energy popping up. Now we are aware of this, it follows straightforwardly from GR that this energy creates an irregular spacetime at small scales. What is however fatal for spacetime and GR is, as in Arkani-Hamed and Seiberg's story, that below the Planck scale the energy is so high that the spacetime not just becomes irregular, but a black hole singularity is created. Spacetime does not exist at all at those scales. Concluding, we do not need humans probing a small region with high energy to destroy spacetime, quantum fluctuations do the job for us, whether we are around or not.

Wheeler gives an additional reason why one cannot hold on to the concept of spacetime at small length scales. Although quantum theory allows us to talk about space (3-geometries) and time separately, we cannot combine them into one spacetime at small length scales since these concepts cannot be simultaneously well-defined (Misner et al., 1973, §43). The best we could do is to talk about a superspace which gives the amplitudes for the superposition of different 3-geometries. But, as Isham (1994) points out, it becomes problematic to understand physics if the causal structure fluctuates quantum mechanically at these length scales (Tong, 2012).

Besides negative arguments, against the fundamentality of spacetime, there 
are also positive arguments for spacetime being emergent. A number of features of GR is often considered to be suggestive of underlying microstructure (Linnemann and Visser, 2018); if one takes GR to be a theory of spacetime, this would suggest that spacetime is emergent. Arguably the strongest such hint arises from black hole thermodynamics. Black holes are specific metric solutions of GR. They can be described by a few macroscopic parameters which turn out to obey laws that are formally analogous to the laws of thermodynamics (Bardeen et al., 1973; Beckenstein, 1972; Kiefer, 2007; Smarr, 1973). We standardly take thermodynamic systems to have a non-trivial underlying microstructure, say a gas of particles obeying statistical mechanics. The idea is then that black hole thermodynamics indicates that a black hole, or even the metric in GR in general, has a non-trivial underlying microstructure. For this to be a conclusive argument, one would have to show that the analogy is exact, that this formal analogy makes black holes thermodynamic systems in all relevant aspects, and that one could not provide a coherent physical interpretation of black hole thermodynamics without inferring underlying microstructure (Dougherty and Callender, 2016; Prunkl and Timpson, 2016; Wallace, 2018a,b). I will not dwell here on the subtleties of that debate; the interested reader is referred to Linnemann and Visser (2018) - and references therein, especially Jacobson (1995), Padmanabhan (2010) and Verlinde (2011) - for a comprehensive appraisal of these hints towards a microstructure underlying GR.

We are forced to give up either General Relativity and spacetime at small length scales, or quantum theory, or both. ${ }^{18}$ The fact that an extrapolation of the strength of the electromagnetic, strong and weak force to smaller scales results in a single intersection around the Planck scale ${ }^{19}$ suggests that quantum theory remains valid at least up to that scale. (It should be admitted though that gravity joins them not much later.) Although the entire state of the art of quantum gravity is still fragmentary, and it is difficult to reach any definite conclusions, this intersection plus the other arguments in this section do strongly suggest that spacetime is not fundamental. Hence, in the remainder of this paper we will focus on theories that assume General Relativity loses out and could use replacement. ${ }^{20}$ As a bonus we hope to solve some mysteries that quantum theory has currently left unsolved.

The classical debate about the status of space(time) started out as a dilemma between substantivalism and relationalism. After transforming this into a trilemma by pointing out the option of emergent spacetime at the start of the paper, we have now narrowed this back down to an overarching dilemma, albeit a new one: relationalism vs. emergent spacetime (and matter). We arrived at this conclusion mainly because arguments from physics suggest favouring emergent

\footnotetext{
${ }^{18}$ Semi-classical gravity would be an exception, as it claims that (a slightly modified version of) GR is true at all scales without having to be quantized. This approach however is not without its problems (Wüthrich, 2005).

${ }^{19}$ This intersection is especially accurate if supersymmetry is imposed.

${ }^{20}$ It should be flagged though that we do not understand how to do standard quantum theory without a background spacetime, although e.g. Quantum Graphity might be a way of avoiding this.
} 
spacetime theories over their effective counterparts (i.e. substantivalism); moreover, emergent spacetime theories fare at least as well as substantivalism against philosophical arguments that target substantivalism.

\section{Questions we hope the emergent spacetime re- search programme will answer}

In this section I will suggest some questions one may want to keep in mind when looking into (the philosophical commitments and consequences of) several versions of emergent spacetime theories. In the next section these questions will return when discussing a case study. It is important to note that most of these questions are opportunities for emergent spacetime theories rather than difficulties. They are left unanswered by non-emergent theories. If emergent spacetime theories fail to answer them, then nothing is lost; if they manage to answer some of them then this provides a substantive advantage.

Is the theory a theory of emergent ST, super-emergent ST, or emergent ST \& M?

What notion of emergence is used, and how does this relate to the notion of emergence within the relationalist approach?

The philosophical notion of emergent properties or entities usually refers to properties or entities that supervene on more fundamental properties and entities without being reducible to them (but see O'Connor and Wong (2012) for an overview of differing definitions). The vague notion of irreducibility employed here can be cashed out in several ways. One option is epistemic irreducibility: the systems under consideration are so complex that we, humans with limited cognitive capacities that we are, are unable to express the emergent properties in terms of the fundamental properties. Here we are more interested in two other versions of emergentism: inter-theoretical and ontological emergentism. The first appears when the vocabulary used in the emergent theory is so different from the vocabulary of the fundamental theory that there is no direct mapping from concepts in one theory to (a combination of) concepts in the other theory. Ontological emergence refers to new primitive entities or properties arising when fundamental entities form a certain pattern. Note that these novel entities are more than just the composition of the fundamental entities; when a new entity emerges it gets added to the list of all fundamental things that exist in the world.

It is controversial whether there are in fact any instances of these latter two types of emergence. ${ }^{21}$ Perhaps our theories of emergent spacetime refer to a broader, common-sense notion of emergence (Matsubara, 2017). Perhaps

\footnotetext{
${ }^{21}$ Crowther (2014) argues that the ontological-epistemological emergence distinction is very unhelpful, particularly in the context of theories of emergent spacetime.
} 
nothing more is meant than that, in some regime, the fundamental degrees of freedom form a pattern which constitutes spacetime. Philosophically speaking this would just be supervenience or constitution. This is the notion of emergent spacetime, if any, that seems to be used by the relationalist. The instantaneous relative particle distances and facts about time intervals between particle configurations that the relationalist has available allow for the construction of a background spacetime that elegantly encodes these facts. This does not imply that spacetime should be attributed a primitive metaphysical status, irreducible to the primitive relational facts. Similarly, emergent spacetime theorists may have no intention of making claims about the relation between two theories: emergence may just refer to the fundamental degrees of freedom only having the right structure to be considered spacetime in one (energy) regime, whereas spacetime does not so arise in other regimes within that same theory.

It is probably fair to say that emergence is a notoriously controversial notion in the philosophy of science. Q2 may then not only be seen as the task of applying philosophical tools to refine our understanding of physical theories, but also (or perhaps mainly) the other way round: theories in which spacetime emerges may provide fruitful casestudies for philosophers to (finally!) get clear on the thorny notion of emergence. Such a science-first approach to the notion of emergence is advocated by Crowther (2014).

What happens to the notion of matter in the regime where spacetime does not emerge?

What additional structure emerges compared to the standard construal of spacetime manifolds?

A. Primitive identities?

B. An orientational structure?

A. Laughlin's Mystery of Mysteries (2005):

Why do the fundamental forces and spacetime have any symmetry at all?

B. Brown's Mystery of Mysteries (2005):

Why are (almost) all forces and spacetime locally Lorentz covariant?

C. Pauli's Mystery of Mysteries (Gardner, 1964/1990):

Why does only the weak force violate Brown's Mystery of Mysteries

by violating parity maximally [and $\mathrm{CP}$ a bit], and not all three forces?

Brown (2005) points out that it seems too much of a coincidence that all three of our fundamental (non-gravitational) interactions (electromagnetism, the strong force and the weak force) share the same symmetries, local Lorentz covariance. As with all such 'coincidences' in the history of science, this suggests an underlying unifying explanation. Laughlin (2005) makes the stronger point that, even if there would be no such universality of symmetry, we would 
still require an explanation of the symmetries of each force separately. Symmetries do not cause dynamics - despite particle physicists adopting such a view when constructing Lagrangian theories such as the Standard Model of Particle Physics - but are a property of dynamics (or spacetime) which stands in need of explanation. In fact, the universality pointed out by Brown is not strictly correct. All three interactions are covariant under the restricted Lorentz group, but only the strong force and electromagnetism are covariant under the full Lorentz group. This is the real mystery, according to Pauli: why does only the weak force violate parity? Hopefully theories of emergent spacetime (and matter) can shed some light on these mysteries, especially those theories in which all dynamics become connected through a single set of fundamental degrees of freedom all governed by the same dynamical law.

Does the emergent spacetime theory explain inertial motion?

In Newtonian physics inertial motion is postulated. Perhaps emergent spacetime theories will tell us why geodesics are the way they are, and, in case of theories in which there is a link between matter and spacetime, it may be explained why matter follows these geodesics. It is often argued that inertial motion is already explained by the geodesic principle in General Relativity, which holds that inertial motion can be derived from Einstein's field equations (Brown, 2005; Ehlers and Geroch, 2004; Geroch and Jang, 1975; Geroch and Weatherall, manuscript; Weatherall, 2017). Whether the geodesic theorem indeed succeeds in removing the need of postulating inertial motion is controversial (Malament, 2009; Sus, manuscript; Tamir, 2012; Yang, 2013), but even if it does succeed a deeper explanation may be gained from certain theories in which not only spacetime but also gravity itself is emergent.

Why does spacetime emerge with $3+1$ dimensions?

\section{A case study: quantum superfluids}

Bain $(2008,2013)$ discusses the emergence of relativistic spacetime from condensed matter systems, specifically quantum liquid toy models such as superfluid Helium-3 and superfluid Helium-4. ${ }^{22}$ For instance, the ground state of superfluid Helium-4 is a Bose-Einstein condensate. The corresponding non-relativistic Lagrangian, on a Neo-Newtonian spacetime (i.e. Galilean spacetime), is:

$$
\mathcal{L}_{4}{ }_{H e}=i \phi^{\dagger} \partial_{t} \phi-\frac{1}{2 m} \partial_{i} \phi^{\dagger} \partial_{i} \phi+\mu \phi^{\dagger} \phi-\kappa\left(\phi^{\dagger} \phi\right)^{2}, i=1,2,3,
$$

\footnotetext{
${ }^{22}$ As pointed out by an anonymous referee, the idea of spacetime emerging from a superfluid/condensate is realised (partially) in group field theory, at least for cosmological spacetimes which can be seen to arise as condensates of the microscopic group field theory 'atoms', in turn representable as spin networks or simplicial structures.
} 
where $m$ is the mass of the Helium- 4 atoms, $\mu$ the chemical potential. One can then obtain an effective field theory for this Lagrangian, valid at low energies, by expanding $\phi$ around the ground state $\left(\phi=\sqrt{\rho} e^{i \theta}, \rho=\rho_{0}+\delta \rho, \theta=\theta_{0}+\delta \theta\right)$, where the relevant low-energy excitations are fluctuations in the phase, $\delta \theta$.

$$
\mathcal{L}_{\text {eff }}=\frac{1}{4 \kappa}\left(\partial_{t} \theta+v_{i} \partial_{i} \theta\right)^{2}-\frac{\rho_{0}}{2 m}\left(\partial_{i} \theta\right)^{2},
$$

such that $\mathcal{L}^{4}{ }_{H e}=\mathcal{L}_{0}\left[\rho_{0}, \theta_{0}\right]+\mathcal{L}_{\text {eff }}[\delta \theta]$, where we have substituted $\delta \theta$ for $\theta$ for the sake of notation and $v$ is the superfluid velocity. $\mathcal{L}_{\text {eff }}$ is formally identical to

$$
\mathcal{L}_{\text {eff }}=\frac{1}{2} \sqrt{-g} g^{\mu \nu} \partial_{\mu} \theta \partial_{\nu} \theta, \mu, \nu=0,1,2,3,
$$

where $g=g(v, \rho, m)$. This Lagrangian now formally describes a massless scalar field in a (3+1)-dimensional curved relativistic spacetime! Relativistic spacetime has emerged from a quantum liquid (living on a non-relativistic spacetime)!

In this emergent spacetime theory we start out with (non-relativistic) spacetime-like fundamental degrees of freedom and matter-like degrees of freedom, a fundamental condensate, living 'on' the former set of degrees of freedom. Matter as we know it then emerges as collective modes of the ground state of the fundamental condensate; in this regime the dynamics of the emergent matter is so that it mimics living in a relativistic spacetime (or in other words, its dynamics are most simply described using a mathematical theory containing a relativistic background). The most straightforward interpretation of this toy model is that we should categorise it as an 'emergent spacetime \& matter' theory (Q1): in a certain regime the (matter-like) fundamental degrees of freedom (which live on spacetime-like degrees of freedom) mimic what we normally consider matter living in what we normally consider (relativistic) spacetime. Although this seems the best choice from the three available types of emergent spacetime theories distinguished earlier, this will not convince a relationalist. A relationalist would maintain that the spacetime-like fundamental degrees of freedom should not be interpreted as fundamental entities, but rather as properties of the matter-like degrees of freedom. Matter and spacetime as we know them then emerge through composite behaviour of these matter-like entities and their spatiotemporal-like properties.

Bain seems to construe the notion of emergence that arises in this example as a combination of ontological and inter-theoretic emergence (Q2). The effective theory is obtained from the fundamental theory by integrating out the high energy degrees of freedom. For this reason, Bain argues, the effective theory is a truly novel theory, as it becomes irreducible to the fundamental theory and is committed to a different ontology (e.g. a relativistic spacetime). It seems strange to interpret this as ontological emergence - that is in the regime where the effective theory is valid it replaces the fundamental theory and its ontology. Although there is no logical contradiction in the ontology (the list of fundamental things) of our actual world changing over time, it seems implausible that a specific combination of fundamental things creates a new fundamental entity or property. Even more implausible is that that primitive (i.e. fundamental) 
thing should disappear again if the world exits the regime in which the effective theory is valid; something that is primitive does, by definition, not depend on other things for it to endure. If, on the other hand, Bain's story is to be interpreted as emergence being a relation between the fundamental and effective theories, this suggests that spacetime is a notion which can only be understood within the effective theory, which we know to be merely a useful tool which is no true representation of the ontology of our actual world. Such eliminativism about spacetime seems too strong. Both the fundamental theory and the effective theories are Lagrangian theories - the effective theory is obtained as an approximation to the fundamental theory. Our original theory already contained spacetime-like degrees of freedom, the only thing that changes is that in the low energy regime these properties (together with the matter-like degrees of freedom) approximately mimic relativistic properties. It would be an exaggeration to claim that the fundamental (and hence the only correct) theory of our world has no grip on the notion of relativistic spacetime.

It seems that a broader notion of emergence is appropriate here. Within a single theory, relativistic spacetime emerges in a certain regime because in that regime the fundamental entities and properties of the theory combine in the right way to approximately mimic what we call relativistic spacetime. No dynamical ontologies, or relations between theories are required. A simple notion of constitution or supervenience suffices.

Whether this spacetime theory explains inertia (Q6) remains undecided since the current toy models fail to fully reproduce General Relativity (see Bain (2008); although multi-component Bose Einstein condensates seem to have a better chance at succeeding (Girelli et al., 2009, 2008; Sidoni, 2011)). It would be too strong to claim that this theory has explained the dimensionality of spacetime (Q7), since this follows from the dimensionality of the background spatiotemporal degrees of freedom which remains unexplained itself (but see Hall's example of quantum Hall liquids which seem to do a better job). A similar story applies to the explanation of (Lorentz) symmetry (Q5). These symmetries somewhat miraculously appear in the effective theory, from the underlying dynamics and the Galilean symmetry of the background spacetime-like degrees of freedom, but this only leaves us with the question of why those degrees of freedom had that symmetry. (It is worth pointing out though that these systems do allow us to learn something about the relative scale of fundamental discreteness vs. Lorentz invariance: a breakdown of the effective hydrodynamics can occur before or after the breakdown of the emergent Lorentz invariance. This can be studied in detail. ${ }^{23}$ ) Nothing is learned when it comes to the question of why only the weak force violates parity, but we would not have expected that much from a mere toy model. Fortunately we do not need to worry about spacetime emerging with primitive identities (Q4), as long as the fundamental degrees of freedom have no primitive identities.

\footnotetext{
${ }^{23}$ I would like to thank an anonymous referee for pointing this out.
} 


\section{Outlook}

In this paper I have argued that the debate between substantivalism and relationalism knows an important third player: theories in which spacetime is an emergent concept. In fact, although the philosophical arguments do not distinguish between emergent spacetime theories and substantivalism, it is modern physics that suggests that substantivalism should be given up in favour of emergent spacetime. I have provided a non-exhaustive list of philosophical questions one should consider when discussing the merits of a theory of emergent spacetime (compared to its opponent in the new, remaining dilemma: relationalism). These questions have been discussed in the context of a superfluid toy model of an emergent spacetime theory. Many questions cannot be answered yet or have an unsatisfactory answer. Future work should discuss whether more mature examples of emergent spacetime theories (see Carlip (2012) for a list) prove more successful in answering these questions, and hence form viable candidates in opposing relationalism.

\section{Acknowledgements}

I would like to thank Dennis Lehmkuhl, Niels Linnemann, Tushar Menon, Oliver Pooley, Carina Prunkl, Simon Saunders, David Wallace and anonymous referees for useful discussions and/or comments on earlier drafts of this paper, and for their generosity with their time. I am grateful for questions and comments from the audience at the OZSW Graduate Conference for Theoretical Philosophy 2014 in Amsterdam. The major part of this paper was written while I was at Magdalen College and Department of Philosophy, University of Oxford, supported by the Arts and Humanities Research Council of the UK and a Scatcherd European Scholarship. I would also like to acknowledge support from the DFG Research Unit "The Epistemology of the Large Hadron Collider" (grant FOR 2063).

\section{References}

H.G. Alexander, editor. The Leibniz-Clarke Correspondence. Manchester: Manchester University Press, 1956/1717. Originally written by Leibniz, G.W. \& Clarke, S. in 1715-16 and published by Clarke, S. in 1717.

Giovanni Amelino-Camelia. Relativity in space-times with short-distance structure governed by an observer-independent (planckian) length scale. International Journal of Modern Physics D, 11:35-60, 2002.

Arkani-Hamed. Messenger lecture 3: Space-time is doomed. what replaces it?, Oct 2010. URL http://www.cornell.edu/video/ nima-arkani-hamed-spacetime-is-doomed.

Frank Arntzenius. Space, Time, \& Stuff. Oxford University Press, 2012. 
J. Bain. Condensed matter physics and the nature of spacetime. In D. Dieks, editor, The Ontology of Spacetime II, chapter 16. Elsevier Science and Technology, 2008.

J. Bain. The emergence of spacetime in condensed matter approaches to quantum gravity. Studies in History and Philosophy of Modern Physics, 44:338$345,2013$.

David John Baker. On spacetime functionalism. Manuscript, 2018. URL http: //philsci-archive.pitt.edu/14301/.

James M. Bardeen, Brandon Carter, and Stephen W. Hawking. The four laws of black hole mechanics. Comm. Math. Phys., 31(2):161-170, 1973.

J. Beckenstein. PhD thesis, 1972.

Gordon Belot. Geometric Possibility. Oxford University Press, 2011.

M. Bronstein. Quantentheorie schwacher gravitationsfelder. Phys. Z. Sowjetunion, 9:140-157, 1936.

Harvey R. Brown. Physical Relativity: Space-time structure from a dynamical perspective. Oxford University Press, 2005.

S. Carlip. Challenges for emergent gravity. arXiv:1207.2504v2, 2012.

Karen Crowther. Appearing Out of Nowhere: The Emergence of Spacetime in Quantum Gravity. PhD thesis, The University of Sydney, 2014. URL arXiv: $1410.0345 \mathrm{v} 1$.

Shamik Dasgupta. Substantivalism vs relationalism about space in classical physics. Philosophy Compass, 10(9):601-624, 2015.

Neil Dewar. Sophistication about symmetries. Forthcoming in The British Journal for the Philosoph of Science, forthcoming.

John F. Donoghue. The effective field theory treatment of quantum gravity. arXiv:1209.3511v1, 2012. URL arXiv:1209.3511v1.

Sergio Doplicher, Klaus Fredenhagen, and John E. Roberts. The quantum structure of spacetime at the planck scale and quantum fields. Commun. Math. Phys., 172:187-220, 1995.

John Dougherty and Craig Callender. Black hole thermodynamics: More than an analogy? Manuscript, 2016. URL http://philsci-archive.pitt.edu/ 13195/.

F. Dowker. Causal sets and the deep structure of spacetime. arXiv:grqc/0508109v1, 2008.

Patrick Dürr. Do gravitational waves carry energy? -critique of a procrustean practice. PhilSci Archive, 2015. 
J. Earman. World Enough and Space-Time: Absolute versus Relational Theories of Space and Time. Cambridge, Massachusetts: MIT, 1989.

J. Earman and J.D. Norton. What price spacetime substantivalism. British Journal for the Philosophy of Science, 38:515-525, 1987.

J. Ehlers and R. Geroch. Equation of motion of small bodies in relativity. Annals Phys., pages 232-236, 2004.

A. Einstein. Time, space, and gravitation. Times (London), 28 November 1919. 13-14. Reprinted as "What is the Theory of Relativity?" in Einstein (1954), Ideas and Opinions. New York: Bonanza Books, 227-232.

M. Gardner. The New Ambidextrous Universe: Symmetry and Asymmetry from Mirror Reflections to Superstrings. Mineola, New York: Dover Publications, Inc, dover 2005 (= third) edition, 1964/1990. revised and retitled in 1990.

R. Geroch and P.S. Jang. Motion of a body in general relativity. Journal of Mathematical Physics, 16:65, 1975.

Robert Geroch and James Owen Weatherall. The motion of small bodies in space-time. arXiv:1707.04222, manuscript. URL arXiv:1707.04222.

F. Girelli, S. Liberati, and L. Sidoni. Emergence of lorentzian signature and scalar gravity. Physical Review D, 79, 2009.

Florian Girelli, Stefano Liberati, and Lorenzo Sidoni. Gravitational dynamics in bose einstein condensates. Physical Review D, 78, 2008.

G.E. Gorelik. Matvei bronstein and quantum gravity: 70th anniversary of the unsolved problem. Phys. Usp, 48:10391053, 2005.

Carl Hoefer. The metaphysics of space-time substantivalism. The Journal of Philosophy, 93(1):5-27, 1996.

Sabine Hossenfelder. Minimal length scale scenarios for quantum gravity. Living Rev. Relativity, 16(2), 2013.

Nick Huggett. Reflections on parity nonconservation. Philosophy of Science, 67 (2):219-241, 2000.

C.J. Isham. Prima facie questions in quantum gravity. In J. Ehlers and H. Friedrich, editors, Canonical Gravity: From Classical to Quantum (Lecture Notes in Physics 434). Berlin: Springer-Verlag, 1994.

Ted Jacobson. Thermodynamics of spacetime: The einstein equation of state. Physical Review Letters, 75(7):1260, 1995.

Claus Kiefer. Quantum Gravity, volume 136. Oxford University Press, 2nd edition, 2007. 
Eleanor Knox. Effective spacetime geometry. Studies in History and Philosophy of Modern Physics, 44(3):346-356, 2013.

Eleanor Knox. Physical relativity from a functionalist perspective. Forthcoming in Studies in History and Philosophy of Modern Physics, 2018.

T. Konopka, F. Markopoulou, and L. Smolin. Quantum graphity. arXiv:0611197v1, 2006.

T. Konopka, F. Markopoulou, and S. Severini. Quantum graphity: a model of emergent locality. arXiv:0801.0861v2, 2008.

R.B. Laughlin. A Different Universe: Reinventing physics from the bottom down. New York: Basic Books, 2005.

Baptiste Le Bihan and Niels Linnemann. Have we lost spacetime on the way?: Narrowing the gap between general relativity and quantum gravity. 2018.

Baptiste Le Bihan and James Read. Duality and ontology. Forthcoming in Philosophy Compass, forthcoming.

D. Lehmkuhl. Spacetime Matters: On Super-Substantivalism, General Relativity, and Unified Field Theories. PhD thesis, Oriel College, University of Oxford, 2009.

Dennis Lehmkuhl. Mass-energy-momentum: Only there because of spacetime? The British Journal for the Philosophy of Science, 62(3):453-488, 2011. doi: 10.1093/bjps/axr003. URL http://dx.doi.org/10.1093/bjps/axr003.

Dennis Lehmkuhl. The metaphysics of super-substantivalism. Noûs, 52(1):2446, 2018. doi: 10.1111/nous.12163. URL https://onlinelibrary.wiley. com/doi/abs/10.1111/nous. 12163.

Niels S. Linnemann and Manus R. Visser. Hints towards the emergent nature of gravity. Studies in History and Philosophy of Science Part B: Studies in History and Philosophy of Modern Physics, 64:1 - 13, 2018. ISSN 13552198. doi: https://doi.org/10.1016/j.shpsb.2018.04.001. URL http://www . sciencedirect.com/science/article/pii/S1355219817302046.

D.B. Malament. A remark about the "geodesic principle in general relativity". http://philsci-archive.pitt.edu/5072/, 2009. URL http:// philsci-archive.pitt.edu/5072/. Version 3.0.

N.C.M. Martens. Parity violation and the reality of space. In Proceedings of the VSNU Student Research Conference, Utrecht, the Netherlands, 21 November 2012, pages 221-224, 2012. URL http://oxford.academia.edu/ NielsMartens/Papers.

Niels C.M. Martens and Dennis Lehmkuhl. Dark matter = modified gravity? scrutinising the spacetime-matter distinction through the modified gravity/ dark matter lens. manuscript. 
Niels C.M. Martens and James Read. Sophistry about symmetries? manuscript.

K. Matsubara. Quantum gravity and the nature of space and time. Philosophy Compass, 12(e12405), 2017.

Tim Maudlin. The essence of spacetime. In PSA: Proceedings of the Biennial Meeting of the Philosophy of Science Association, Volume Two: Symposia and Invited Papers, 1988.

Tim Maudlin. Buckets of water and waves of space: Why spacetime is probably a substance. Philosophy of Science, 60:183-203, 1993.

C.A. Mead. Possible connection between gravitation and fundamental length. Phys. Rev., 135:B849B862, 1964.

C.A. Mead. Observable consequences of fundamental-length hypotheses. Phys. Rev., 143:990-1005, 1966.

C.W. Misner, K.S. Thorne, and J.A. Wheeler. Gravitation. New York: W.H. Freemand and Company, 1973.

Jill North. A new approach to the relational-substantival debate. In Oxford Studies in Metaphysics (forthcoming). 2017.

T. O'Connor and H.Y. Wong. Emergent properties, 2012. URL http://plato. stanford.edu/entries/properties-emergent/\#EpiEme. Stanford Encyclopedia of Philosophy. Original version from 2002.

Thanu Padmanabhan. Thermodynamical aspects of gravity: New insights. Rept. Prog. Phys., 73:046901, 2010.

A. Peres and N.. Rosen. Quantum limitations on the measurement of gravitational fields. Phys. Rev., 118:335-336, 1960.

O. Pooley. The Reality of Spacetime. Dphil thesis, Oriel College, University of Oxford, 2002.

O. Pooley. Handedness, parity violation and the reality of space. In Katherine Brading \& Elena Castellani, editor, Symmetries in Physics: Philospohical reflections, pages 250-280. Cambridge University Press, 2003. See also Pooley (2002).

O. Pooley. The reality of spacetime. book manuscript.

Oliver Pooley. Substantivalist and relationalist approaches to spacetime. In Robert Batterman, editor, The Oxford Handbook of Philosophy of Physics. Oxford University Press, 2013.

Carina Prunkl and Christopher Timpson. Black hole entropy is entropy (and not information). Manuscript, 2016. 
James Read. Functional gravitational energy. The British Journal for the Philosophy of Science, page axx048, 2018. doi: 10.1093/bjps/axx048. URL http://dx.doi.org/10.1093/bjps/axx048.

C. Rovelli and F. Vidotto. Covariant Loop Quantum Gravity: An Elementary Introduction to Quantum Gravity and Spinfoam Theory. Cambridge University Press, 2014. URL http://www.cpt.univ-mrs.fr/ rovelli/ IntroductionLQG.pdf.

Robert Rynasiewicz. Absolute versus relational space-time: An outmoded debate? Journal of Philosophy, 93(6):279-306, 1996.

Jonathan Schaffer. Spacetime the one substance. Philosophical Studies, 145: 131-148, 2009.

N. Seiberg. Emergent spacetime. In Rapporteur talk at the 23rd Solvay Conference in Physics, 2005.

Lorenzo Sidoni. Emergent gravitational dynamics from multi-bec hydrodynamics? Physical Review D, 83, 2011.

L. Smarr. Phys. Rev. Letters, 30:71-73, 1973.

Hartland S. Snyder. Quantized space-time. Physical Review, 71:38-41, 1947.

A. Sus. On the explanation of inertia. manuscript.

M. Tamir. Proving the principle: Taking geodesic dynamics too seriously in einstein's theory. Studies in History and Philosophy of Modern Physics, 43: 137-154, 2012.

D. Tong. Lecture notes string theory, 2012. URL http://www.damtp.cam.ac . uk/user/tong/string/string.pdf.

Erik P. Verlinde. On the origin of gravity and the laws of newton. Journal of High Energy Physics, 4:1-27, 2011.

David Wallace. The case for black hole thermodynamics part i: Phenomenological thermodynamics. Studies in History and Philosophy of Science Part B: Studies in History and Philosophy of Modern Physics, 64:52 - 67, 2018a. ISSN 1355-2198. doi: https://doi.org/10.1016/j.shpsb.2018.05.002. URL http: //www.sciencedirect.com/science/article/pii/S1355219817301661.

David Wallace. The case for black hole thermodynamics, part ii: statistical mechanics. Forthcoming in Studies in the History and Philosophy of Modern Physics, 2018b.

James Owen Weatherall. Conservation, inertia, and spacetime geometry. Studies in the History and Philosophy of Modern Physics, 2017. 
Christian Wüthrich. To quantize of not to quantize: Fact and folklore in quantum gravity. Philosophy of Science, 72(5):777-788, 2005.

S. Yang. On the geodesic hypothesis in general relativity. Communications in Mathematical Physics, art. Digital Object Identifier (DOI) 10.1007/s00220013-1834-7, 2013. 